\title{
LOCALISATION \\ DE TRYPANOSOMA (NANNOMONAS) CONGOLENSE Broden, 1904 (KINETOPLASTIDA, TRYPANOSOMATIDAE) DANS LE PROBOSCIS \\ DE GLOSSINA MORSITANS MORSITANS Westwood, 1850 (DIPTERA, GLOSSINIDAE)
}

\author{
E. LADIKPO*, C. SEUREAU**
}

RÉSUMÉ. Des coupes transversales de la trompe de Glossines ont été effectuées après une infestation expérimentale précoce et unique. Suivant les niveaux du proboscis, les Trypanosomes. sont localisés dans les zones latérales, dorso-ventrales ou latéro-ventrales du canal alimentaire. Ils peuvent aussi occuper toute la périphérie du canal. Ces localisations influent sur la transmission et l'épidémiologie de la trypanosomiase.

Mots-clés : Trypanosoma congolense, Glossina morsitans. Localisation. Proboscis.

Localization of Trypanosoma (Nannomonas) congolense Broden, 1904 (Kinetoplastida, Trypanosomatidae) in the proboscis of Glossina morsitans morsitans Westwood, 1850 (Diptera, Glossinidae).

SUMMARY. Transverse sections of the proboscis of G. m. morsitans infected with T. (N.) congolense initiated after a single infective blood meal were studied. Trypanosomes were located in the lateral, dorso-ventral and latero-ventral regions of the food canal. Parasites were also found around the whole of the circumference of the canal. Such localizations influence the transmission of trypanosomes and the epidemiology of trypanosomiasis.

Key-words: Trypanosoma congolense. Glossina morsilans. Localization. Proboscis.

A la fin de son cycle évolutif chez Glossina morsilans, Trypanosoma (Nannomonas) congolense occupe des localisations particulières dans la trompe de la Glossine. Ces localisations peuvent entraîner des modifications du comportement de la mouche infectée comme la fréquence accrue des coups de trompe par suite

* Institut d'Élevage et de Médecine Vétérinaire des Pays Tropicaux, 10, rue Pierre-Curie, F 94704 Maisons Alfort Cedex.

** Laboratoire d'Histophysiologie fondamentale et appliquée, Université de Paris VI, 12, rueCuvier, $F 75005$ Paris.

Avec l'aide technique de A. Anglo.

Accepté le 20 novembre 1987. 
du mauvais fonctionnement des mécano-récepteurs du labre ou l'augmentation de la durée de prise de sang liée au blocage partiel de la lumière du labre. Ces aspects particuliers de la biologie des Glossines infectées jouent un rôle important dans l'épidémiologie des trypanosomiases (Molyneux, 1980 ; Livesey et al., 1980 ; Molyneux et Jenni, 1981).

Entre 17 et 40 jours après l'infestation expérimentale, les Trypanosomes sont libres dans la lumière du canal alimentaire dans la zone de l'hyoïde (partie proximale du bulbe de la trompe ou zone 11 de Jobling (1933) (fig. 1). Dans la zone du subhyoïde (fig. 1, zone 12), ils sont fixés dans les régions dorsales et ventrales du canal. Enfin, dans celle des sensilles LC1 du labre (fig. 1, zones 15 et 16), ils sont fixés sur les parois latérales du canal alimentaire (Thévenaz et Hecker, 1980). De plus, la densité des Trypanosomes est plus importante dans la partie proximale du proboscis que dans la partie distale (Clarke, 1965, Thévenaz et Hecker, 1980 ; Jefferies et al., 1987).

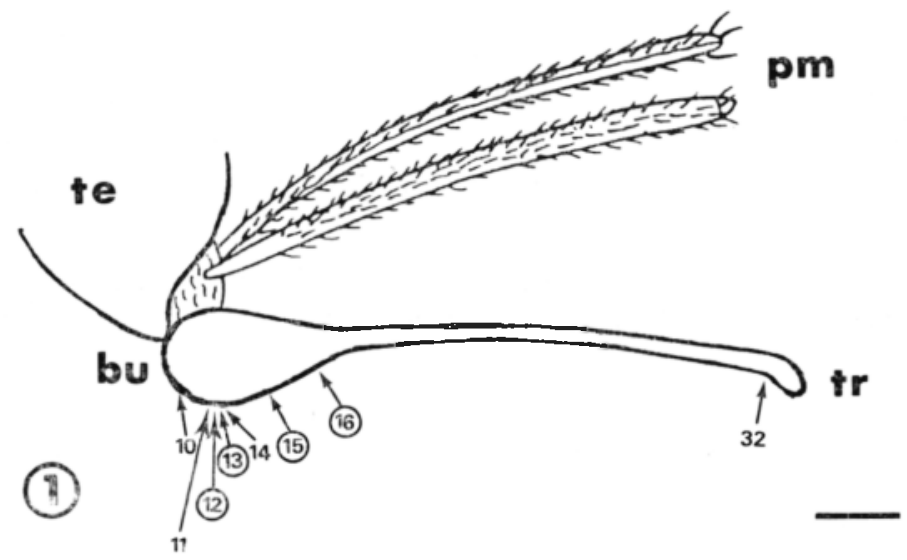

Fig. 1. - Schéma de la trompe de la Glossine. Les flèches numérotées indiquent les différents niveaux cités dans le texte (d'après Jobling, 1933). (12), (13), (15) et (19) $=$ niveaux correspondant respectivement aux figures $2,5,3$ et 4 . bu $=$ bulbe de la théca, $\mathrm{pm}=$ palpes maxillaires, te $=$ tète, $\operatorname{tr}=$ trompe. La barre représente $0,2 \mathrm{~mm}$.

Dans le but de préciser la localisation des Trypanosomes (T. (N.) congolense, souche EATRO 325), des Glossines (G. m. morsitans, souche du Zimbabwe) ont été infestées avec un seul repas pris moins de 24 heures après leur éclosion. Les mouches ont été fixées 21,60 et 72 jours après cette infestation unique. Les trompes ont été incluses dans un mélange épon-araldite et des coupes semi-fines transversales ont été réalisées sur une longueur correspondant approximativement aux niveaux 10 à 32 de Jobling (1933), et examinées au microscope photonique après coloration au bleu de toluidine ( $f i g .2$ à 5 ). Nos observations confirment la position dorso-ventrale des Trypanosomes dans le canal alimentaire au niveau 12, et leur position latérale au niveau 15 (Thévenaz et Hecker, 1980) (fig. 2 et 3). En revanche, à partir du niveau 16, la plupart des Trypanosomes sont fixés en région latéro- 

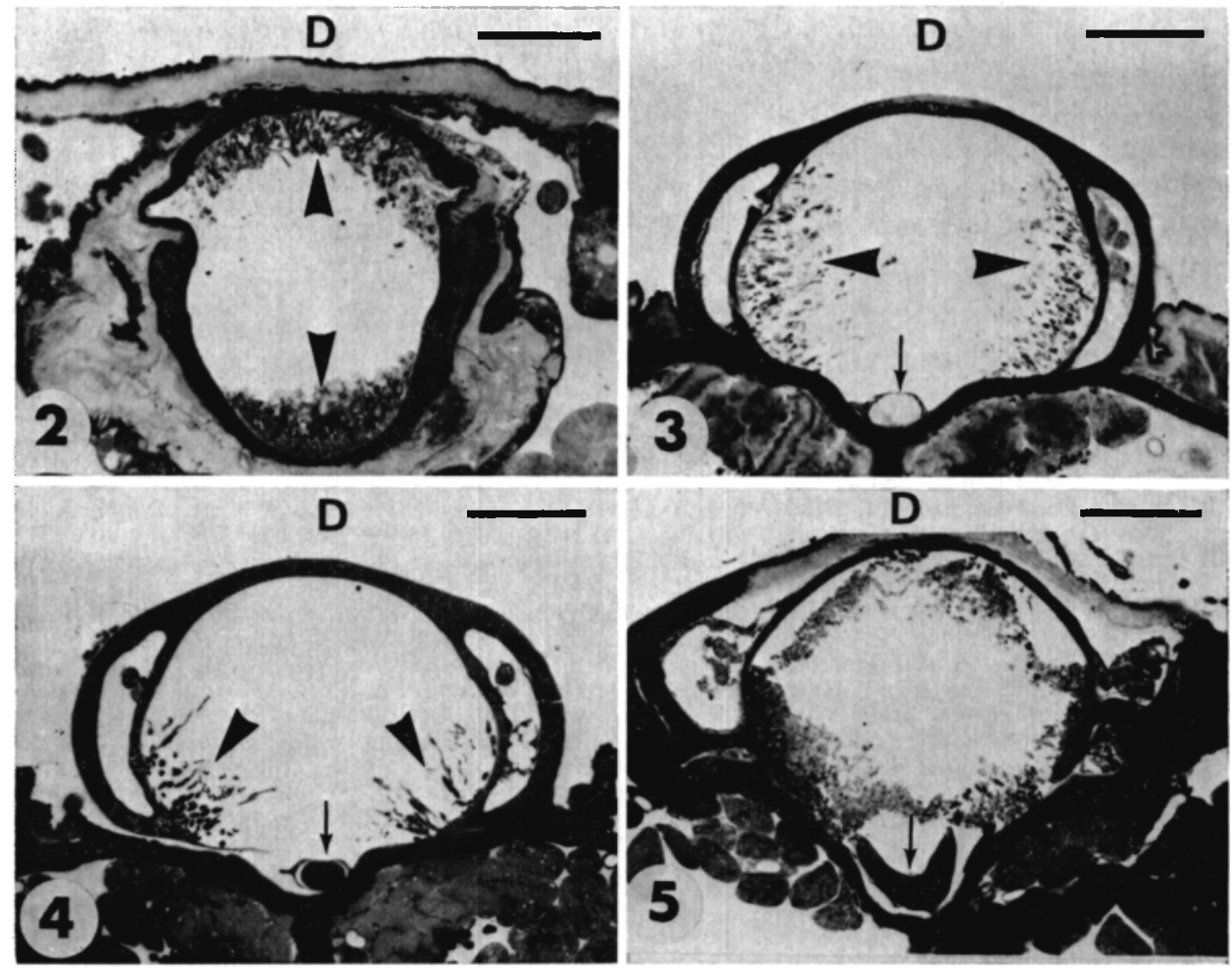

FIG. 2 à 5. - Coupes transversales de la trompe de Glossina morsitans morsilans infectée par Trypanosoma (Nannomonas) congolense (stade épimastigote) ( $\mathrm{D}=$ côté dorsal, grandes flèches $=$ Trypanosomes, petites flèches $=$ hypopharynx). Barre $=50 \mu \mathrm{m}$.

Fig. 2 : Niveau (12.) Trypanosomes en position dorso-ventrale. 72 jours après l'infestation expérimentale (I. E.).

Fig. 3 : Niveau (15). Localisation latérale des Trypanosomes. 72 jours après I. E.

Fig. $t$ : Niveau (16). Localisation latéro-ventrale. 72 jours après I. E.

Fig. 5 : Niveau (13). Trypanosomes sur toute la paroi interne du canal alimentaire. 60 jours après I. $\mathrm{E}$.

ventrale (fig. 4). Aux niveaux 13 et 14, nous observons une localisation qui n'avait pas été signalée auparavant: les parasites sont fixés sur toute la paroi interne du canal alimentaire et quelquefois libres dans la lumière ( fig. 5 ).

Si la densité des Trypanosomes apparaît bien importante dans la zone proximale du proboscis (niveaux 12,13 et 14), elle diminue au fur et à mesure que l'on s'éloigne du bulbe, comme cela avait été observé par les autres auteurs.

Rappelons que les coupes ont été réalisées 21,60 et 72 jours après un seul repas infestant pris moins de 24 heures après l'éclosion des Glossines. La durée moyenne de vie de Glossina morsitans étant d'environ trente jours (pour les mâles) et soixante jours pour les femelles (Pollock, 1982), nos observations montrent qu'une fois infestés, les Insectes-vecteurs demeurent bien infectieux pendant toute la durée de leur vie. 
Ces aspects particuliers du cycle de Trypanosoma (Nannomonas) congolense chez Glossina morsitans morsitans témoignent de la complexité des relations spatiotemporelles entre le parasite et son hôte et des conséquences qui découlent de ces relations complexes dans les domaines de la transmission et de l'épidémiologie des trypanosomiases animales. Ces relations expliquent aussi, en partie, les difficultés souvent rencontrées dans les campagnes de lutte contre ces maladies parasitaires.

\title{
BIBLIOGRAPHIE
}

Clarke J. E. : Trypanosome infections in the mouthparts of Glossina morsitans Westw.: a correlation between extent of labral infection and invasion of the hypopharynx. Ann. Trop. Med. Parasitol., 1965, 59, 235-239.

Jefferies D., Helfrich M. P., Molyneux D. H. : Cibarial infections of Trypanosoma vivax and T. congolense in Glossina. Parasitol. Res., 1987, 73, 289-292.

Jobling B. : A revision of the structure of the head, mouthpart and salivary glands of Glossina palpalis Rob-Desv. Parasitology, 1933, 24, 449-490.

Livesey J. L., Molyneux D. H., Jenni L. : Mechanoreceptor-trypanosome interactions in the labrum of Glossina: fluid mechanisms. Acta Tropica, 1980, 37, 151-161.

Molyneux D. H. : Host-trypanosome interactions in Glossina. Insect. Sci. Appl., 1980, 1, 39-46.

MolyneuX D. H., Jenni L. : Mechanoreceptors, feeding behaviour and trypanosome transmission in Glossina. Trans. Roy. Soc. Trop. Med. Hyg., 1981, 75, 160-162.

Pollock J. N. : Manuel de lutte contre la mouche tsé-tsé, 3 volumes, 564 p. Organisation des Nations-Unies pour l'Alimentation et l'Agriculture, Rome, 1982.

Thévenaz Ph., Hecker H. : Distribution and attachment of Trypanosoma (Nannomonas) congolense in the proximal part of the proboscis of Glossina morsitans morsitans. Acta tropica, $1980,37,163-175$.

\section{CORRIGENDUM}

\section{TRYPANOSOMA JOHNBAKERI NOMEN NOVUM POUR TRYPANOSOMA BAKERI MILTGEN ET LANDAU, 1982}

\author{
F. MILTGEN, I. I,ANDAU
}

Miltgen et Landau (Ann. Parasitol. Hum. Comp., 1982, 57, 423) décrivent un trypanosome nouveau, qu'ils nomment Trypanosoma bakeri, dans le sang d'un Psillacula roseata de Thaïlande et le tube digestif de Culicoides nubeculosus d'élevage. Ce taxon étant déjà utilisé par Chatterjee et Ray pour Trypanosoma avium bakeri, parasite de Pycnonotus jocosus, en Inde, nous renommons le parasite de Psiltacula roseala, Trypanosoma johnbakeri. 